\title{
Kinetic interfaces of patchy particles
}

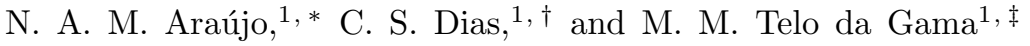 \\ ${ }^{1}$ Departamento de Fúsica, Faculdade de Ciências, \\ Universidade de Lisboa, P-1749-016 Lisboa, Portugal, \\ and Centro de Física Teórica e Computacional, Universidade de Lisboa, \\ Avenida Professor Gama Pinto 2, P-1649-003 Lisboa, Portugal
}

\begin{abstract}
We study the irreversible adsorption of patchy particles on substrates in the limit of advective mass transport. Recent numerical results show that the interface roughening depends strongly on the particle attributes, such as, patch-patch correlations, bond flexibility, and strength of the interactions, uncovering new absorbing phase transitions. Here, we revisit these results and discuss in detail the transitions. In particular, we present new evidence that the tricritical point, observed in systems of particles with flexible patches, is in the tricritical directed percolation universality class. A scaling analysis of the time evolution of the correlation length for the aggregation of patchy particles with distinct bonding energies confirms that the critical regime is in the Kardar-ParisiZhang with quenched disorder universality class.
\end{abstract}

PACS numbers: 82.70.Db,05.70.Ln,05.70.Fh,68.08.De,68.15.+e

\section{INTRODUCTION}

The nonequilibrium evolution of growing interfaces has attracted many experimental and theoretical studies 115. One of the most popular theoretical approaches considers kinetic discrete models to describe particle aggregation on substrates. Albeit simple, these models are expected to contain the relevant physics [16 18. In particular, the ballistic deposition model (BD) [19, 20], is considered the prototype for irreversible aggregation on substrates. In BD, the growth is driven solely by the sequential addition of particles to the aggregate, which stick to the first particle they touch, without subsequent rearrangement. From the simple rules of ballistic deposition a complex structure emerges with a nontrivial porous bulk structure (see e.g., Refs. [16, 19, 21]) and a kinetically rough interface in the Kardar-Parisi-Zhang universality class [16, 22.

Inspired by recent advances in the production of patchy particles we have proposed a stochastic model to study their aggregation on substrates [23], which in the limit of advective mass transport is a generalized version of BD. Patchy particles are colloids with functionalized surfaces, with new features such as selective and directional particle-particle interactions, control over the valence, and the possibility of forming permanent electrical dipoles [24 31]. Studies of the irreversible aggregation on substrates reveal a nontrivial dependence of the bulk and surface properties on the mechanism of mass transport [32], on the strength of the patch-patch interactions [33, 34, and on the spatial-patch distribution [35].

Here, we focus on the scaling properties of the growing interface in the limit of advective mass transport.

\footnotetext{
* nmaraujo@fc.ul.pt

$\dagger$ csdias@fc.ul.pt

$\ddagger$ mmgama@fc.ul.pt
}

In this limit, we have found new absorbing phase transitions depending on the patch-spatial arrangement 35. and a crossover in the universality class of the interface depending on the relative strength of the patch-patch interactions 34. These findings have been discussed previously in the context of functional colloids. Here we revisit these transitions and investigate their scaling properties in the framework of kinetic discrete models of interfacial growth.

The paper is organized in the following way. In Sec. II we describe the model and recall some definitions. The two absorbing transitions are discussed in Secs. III and IV. The crossover of the universality class of the interface is discussed in Sec. V] Some final remarks and future perspectives are provided in Sec.VI

\section{MODEL}

In the ballistic deposition model [16, 18, 36] particles are sequentially released from a position above the interface, chosen uniformly at random, and move vertically towards the substrate sticking irreversibly to the first particle they touch. To account for the directionality of the interactions, the excluded volume interaction between particles, and the short-ranged patch-patch attraction, characteristic of patchy colloids, we proposed a generalized version of this model in Refs. 23, 32, which we describe below.

To access larger-system sizes, let us consider a twodimensional system of patchy particles (disks) of unit diameter $\sigma$ with an initially empty flat (linear) substrate of length $L$. As in the ballistic deposition model, we iteratively generate a horizontal position, chosen uniformly at random above the interface, to release a particle and follow its ballistic trajectory downwards until the particle collides either with the substrate or with another particle. Collisions with the substrate always result on adsorption of the particle at the collision point with a 


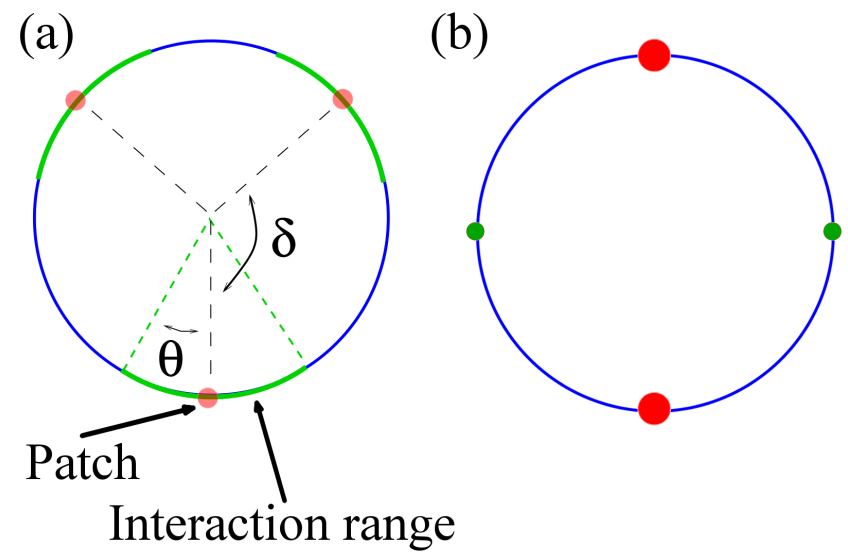

FIG. 1. (a) Patchy particle with three patches (red) on the surface and their interaction range $\theta$ (green). The spatial arrangement of the patches is described by an opening angle $\delta$, in units of $\pi \mathrm{rad}$, from the center of the two adjustable patches to the center of the reference one. (b) Four-patch particle with patches of two types: two $A$-patches on the poles and two $B$-patches along the equator.
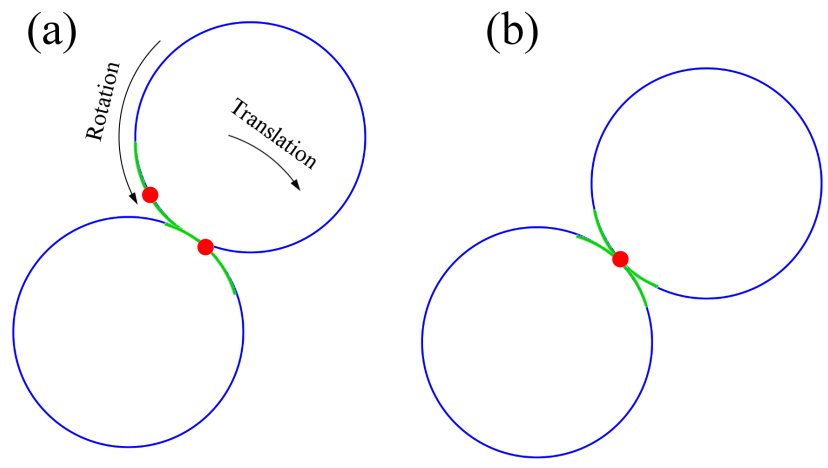

FIG. 2. (a) Successful binding between two patches occurs when their interaction ranges partially overlap, in which case a bond is established aligned along the two patches. Since the particle position and orientation in the aggregate is considered irreversibly fixed, the alignment of the new binding patches results solely from the rotation and translation of the incoming particle, as shown in (b).

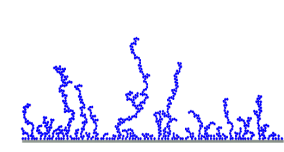

(a)

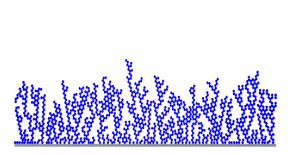

(b)

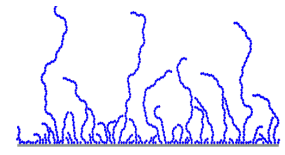

(c)
FIG. 3. Snapshot of networks of patchy particles for different values of $\delta$ : (a) $0.4 \pi$, (b) $2 \pi / 3$, and (d) $0.85 \pi$.

\section{PATCH-PATCH CORRELATIONS}

The dependence of the interface of patchy particle aggregates on the spatial arrangement of the patches was studied in Ref. [35. In that study we considered threepatch particles and investigated the dependence on the opening angle, showing that growth is suppressed below and above a minimum and a maximum opening angles, with two absorbing phase transitions between thick and thin adsorbed film regimes. In this section, we investigate the nature of those transitions.

As shown schematically in Fig. 1(a), the particles are disks with three patches: one reference patch and two adjustable ones. The spatial arrangement of the patches is characterized by the opening angle $\delta$, from the reference patch to the adjustable ones. For simplicity, we define the units of $\delta$ as $\pi \mathrm{rad}$. As the particles are sequentially added, a network of patchy particles grows away from the substrate (see Fig. 3) but, its growth is only sustained for $\delta_{\min }<\delta<\delta_{\max }$.

For $\delta<\delta_{\min }$, the angle between the patches is such that all patches are in the same hemisphere. Thus, the patches of particles in the aggregate are pointing most likely towards the substrate and are not accessible to new incoming particles. When there are no more patches available to establish new bonds, the growth is suppressed. A systematic finite-size study for the thresh- 


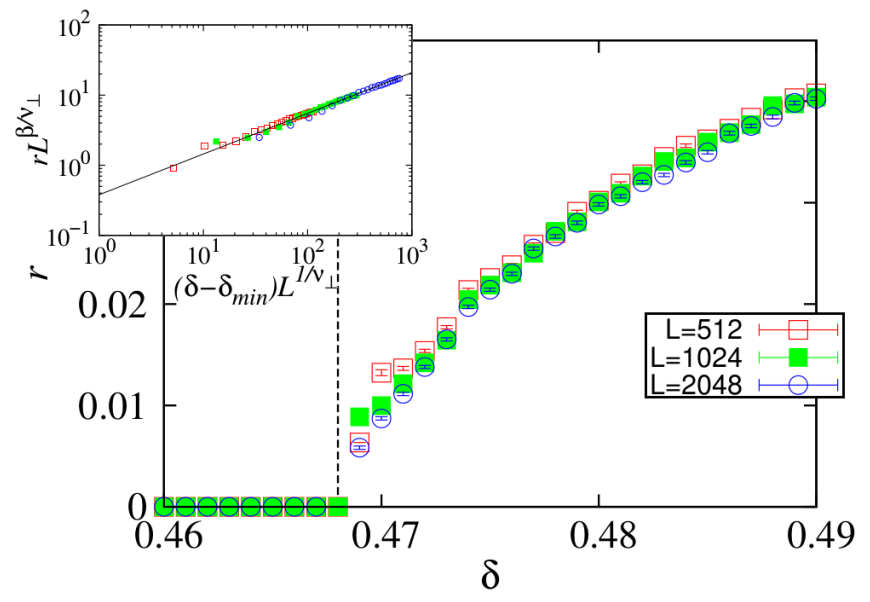

FIG. 4. Fraction $r$ of successful binding attempts in the stationary state as a function of $\delta$, for three different substrate lengths $L$, namely, 512, 1024, and 2048. The inset shows the finite-size scaling in logarithmic scale using the exponents of the directed percolation universality class. We considered $\beta_{\mathrm{DP}}=0.58$ and $\nu_{\mathrm{DP}}=0.73$. Results are averages over $\{400,200,100\}$ samples for $L=\{512,1024,2048\}$.

old value gives $\delta_{\min }=0.468 \pm 0.001$ [35]. This threshold is above that expected from purely geometrical arguments, revealing strong collective effects 35 . To analyze the transition to the absorbing state at $\delta_{\min }$, we define as the order parameter $r$ the fraction of successful binding attempts. Figure 4 , shows the dependence of $r$ on $\delta$ for different system sizes. Clearly, in the thermodynamic limit, $r$ vanishes at $\delta_{\min }$ and it grows continuously with $\delta$. As shown in the inset, a data collapse is obtained for the finite-size scaling consistent with the directed percolation (DP) universality class in two dimensions [18, 40, 41.

For $\delta>\delta_{\max }$, the distance between the adjustable patches is such that, if one particle binds to one of these patches it shields the access of a new particle to the other. Consequently, branching is suppressed and only chains grow away from the substrate. These chains are locally tilted and their growth direction (given by the available patches), fluctuates while growing. Eventually, the growing tip of the chain points down and its growth is suppressed. The absorbing state occurs when all tips are either pointing down or covered by other chains. From geometrical arguments one expects $\delta_{\max }=5 / 6$, a value that was numerically confirmed 35 . Figure 5 shows the dependence of $r$ on $\delta$ close to this second transition. By contrast to the transition at $\delta_{\min }$, at $\delta_{\max }$ the transition is discontinuous and the growth rate jumps at the threshold. In the inset of Fig. 5 it is clear that the jump does not vanish in the thermodynamic limit, discarding the existence of strong finite-size effects.

For $\delta_{\min }<\delta<\delta_{\max }$ a ramified network of patchy particles grows from the substrate in a sustained way. In the stationary state, the interface is always in the Kardar-Parisi-Zhang universality class 22, 35, as was observed for isotropic sticking particles [17, 42]. How-

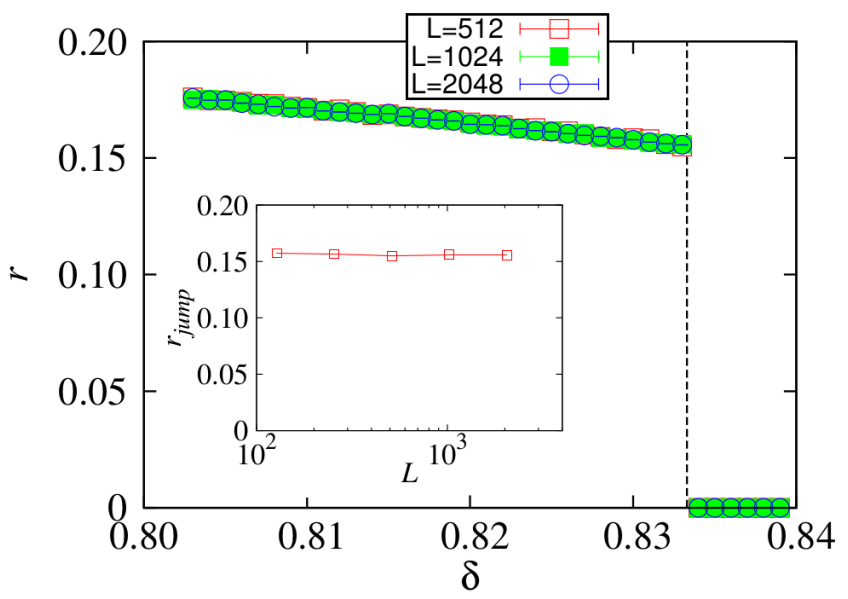

FIG. 5. Fraction $r$ of successful binding attempts in the stationary state as a function of $\delta$, for three different substrate lengths $L$, namely, 512, 1024, and 2048. The inset shows the dependence of the size of the jump on the system size $L$. Results are averages over $\{1600,800,400,200,100\}$ samples for $L=\{128,256,512,1024,2048\}$.

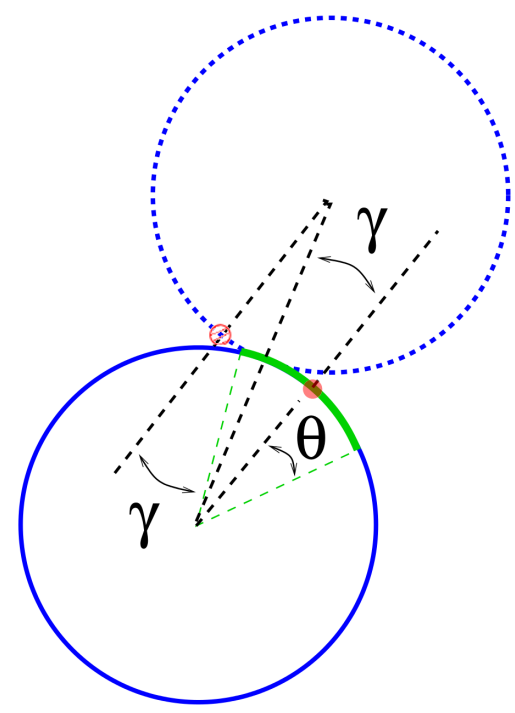

FIG. 6. Non-optimal binding between two patchy particles where the bond orientation deviates from the optimal orientation by an angle $\gamma$. For simplicity, we consider the same deviation for both patches and that the sense of rotation is always from the center of the patch to the point of collision.

ever, for patchy particles the saturation roughness shows a non-monotonic dependence on $\delta$, with a minimum at $\delta=3 / 2$. This minimum occurs when the three patches are equidistant, which favors branching and consequently leads to a decrease of the roughness. 


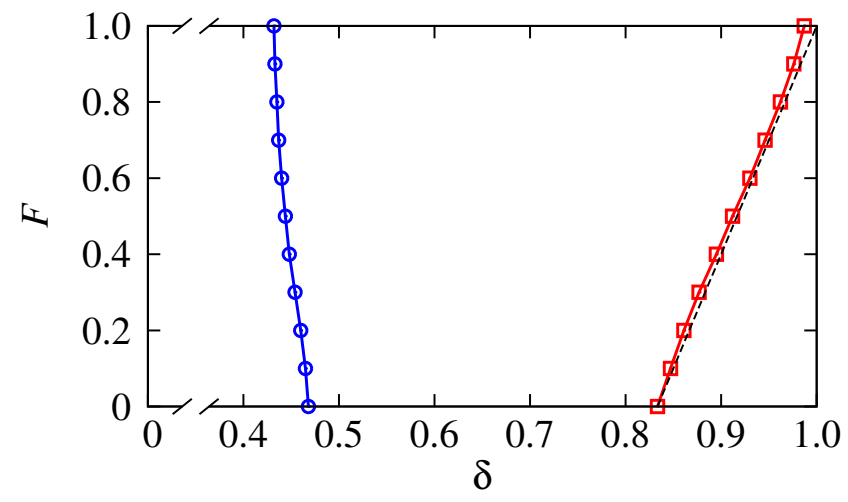

FIG. 7. Phase diagram in the space of flexibility $(F)$ and opening angle $(\delta)$. The solid lines correspond to the lower $\left(\delta_{\min }\right)$ and upper $\left(\delta_{\max }\right)$ thresholds. The data points are extrapolations to the thermodynamic limit from the size dependence of the thresholds. The dashed line is the theoretical prediction for $\delta_{\max }$ based on geometrical arguments.

\section{BOND FLEXIBILITY}

For simplicity, in the previous section the position of the incoming particle is adjusted such that the center of the colloids and of their patches is perfectly aligned. However, in reality, one expects some degree of flexibility around this optimal orientation 37, 43. A simple strategy to account for flexibility was proposed in Ref. 35, which takes advantage of the stochastic nature of our model. The idea is still to consider rigid and irreversible bonds but, at a binding event, the orientation of the bond deviates by an angle $\gamma$ from the optimal orientation (see Fig. 6). The value of $\gamma$ is drawn randomly from a Gaussian distribution of zero mean and dispersion $F \theta$, where $F$ is the flexibility, truncated at $F \theta$. The sense of rotation of $\gamma$ is always from the center of the patch to the point of collision.

Results for different values of the flexibility are summarized in the diagram shown in Fig. 7. The active region does widen with the flexibility but there is always a lower and an upper thresholds. For the range of flexibilities considered, the transition at $\delta_{\min }$ is always continuous and in the DP universality class, as shown in Fig. 8 The threshold $\delta_{\text {min }}$ decreases with $F$ but it is always significantly higher than $1 / 3$, the one predicted from purely geometrical considerations.

For the second transition, the threshold $\delta_{\max }$ increases with $F$ and converges towards unity at large flexibilities. In fact, the threshold value is well approximated by,

$$
\delta_{\max }(F)=\delta_{\max }(0)+\frac{F \theta}{\pi},
$$

obtained from geometrical arguments [35], corresponding to the dashed line in Fig. 7. The nature of the transition also changes with $F$. Figure 9 (a) shows the dependence of the order parameter $r$ on $\delta$ close to the second transition, for three different values of $F$. The numerical

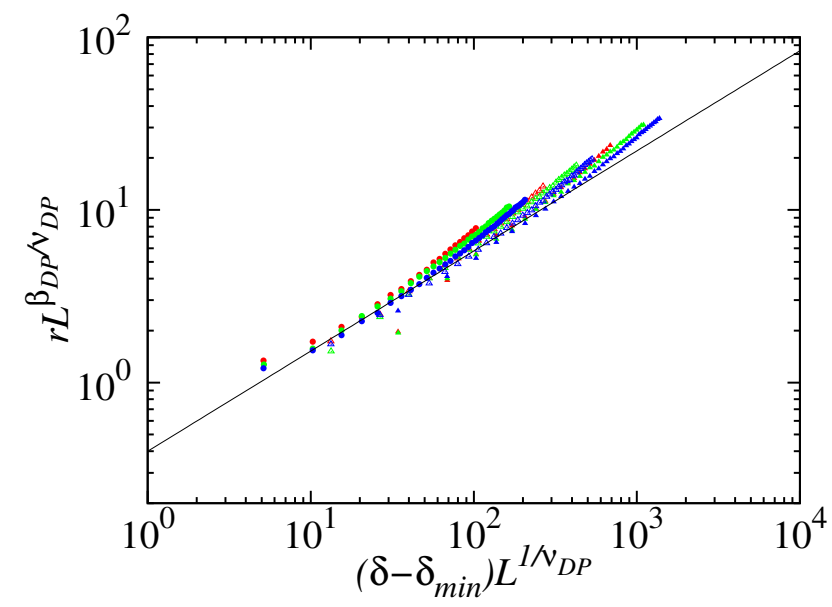

FIG. 8. Finite-size scaling of the order parameter $r$ for different values of the flexibility, $F=\{0.2,0.4,0.6\}$, system size, $L=\{512,1024,2048\}$, averaged over $\{400,200,100\}$ samples. We considered $\beta_{\mathrm{DP}}=0.58$ and $\nu_{\mathrm{DP}}=0.73$ consistent with the DP universality class in two dimensions.
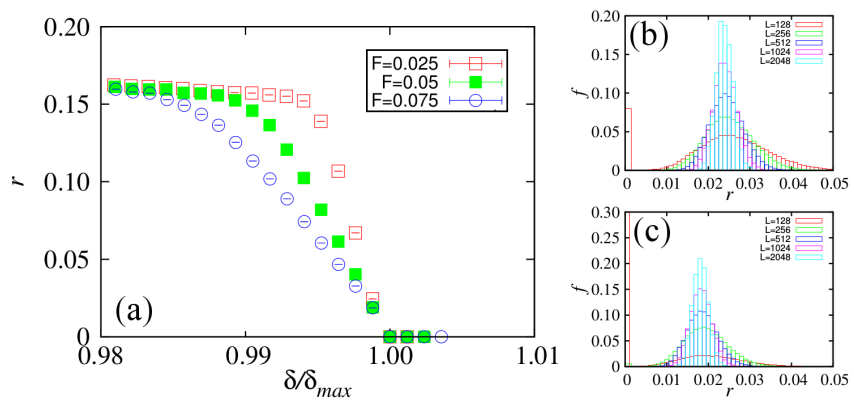

FIG. 9. (a) Order parameter $r$ as a function of $\delta / \delta_{\max }$, for $F=\{0.025,0.05,0.075\}, L=2048$ and averaged over 100 samples. (b) and (c) are the histograms of the order parameter at the threshold $\delta_{\max }$ for $F=0.025$ and $F=0.075$, respectively, showing that the jump does not vanish with the system size. Results are averaged over $\{160000,80000,40000,20000,10000\}$ samples for $L=$ $\{128,256,512,1024,2048\}$.

results suggest that even for $F=0.075$, the transition is still discontinuous (see also the histogram of the order parameter in Fig. 9 (b) and (c)) but the size of the jump decreases as $F$ increases. For $F>0.2$ the numerical results clearly suggest a continuous transition in the DP universality class (see Fig. 10p. The inset of Fig. 10 suggests that the size of the jump decreases with the distance to $F=0.1$ as a power law with exponent 0.25 . This suggests, in turn, that the nature of the transition changes from discontinuous to continuous at a tricritical flexibility $F_{\mathrm{tc}} \approx 0.1$, in the tricritical directed percolation universality class [44, 45]. 


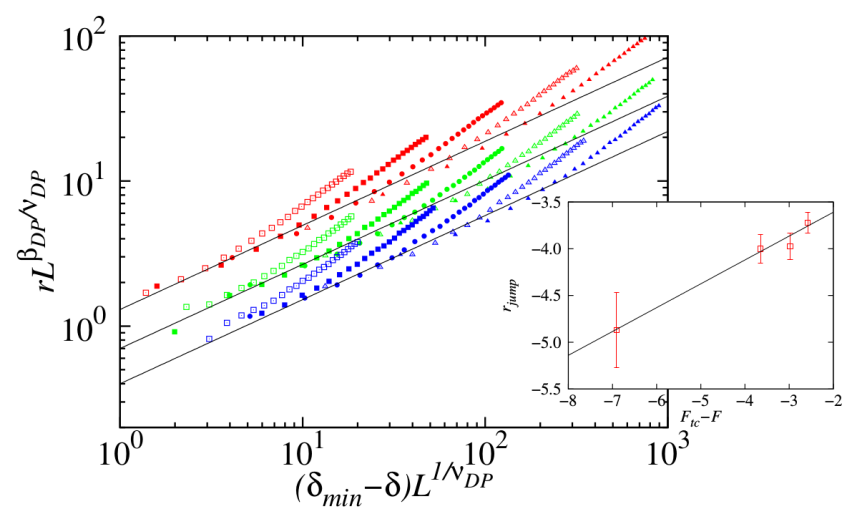

FIG. 10. Finite-size scaling of the order parameter $r$ for $F=\{0.2,0.4,0.6\}$, and three different substrate lengths $L=\{512,1024,2048\}$, averaged over $\{400,200,100\}$ samples. The inset shows the dependence of the size of the jump $r_{\text {jump }}$ on the distance to the tricritical flexibility $F_{\mathrm{tc}}$, for $F<0.1$ and $F_{\mathrm{tc}}=0.1$. The solid line is a guide to the eye scaling as $\left(F_{\mathrm{tc}}-F\right)^{b}$, with $b=0.25$.

\section{WEAK AND STRONG BONDS}

Patchy particles with distinct patch-patch interactions yield interesting bulk properties at equilibrium [46 55 . and novel nonequilibrium interfaces [33, 34. The typical strategy is to consider $2 A n B$ particles, with two strong $A$ - and $n$ weak $B$-patches. The $A$-patches are in the poles, while the $B$-patches are equally spaced along the equator (see Fig[1 (b)). A generalization of our model to account for these two energy scales was proposed in Refs. [33, 34, based on dissimilar binding probabilities that can be formally related to the activation energy of binding. At a collision event with partial overlap between the interaction range of two patches $i$ and $j$, the binding is successful with a binding probability $P_{\mathrm{ij}}$ that depends on the type of patch pair $i j$. In particular, since $A$-patches are stronger than $B$-patches, we consider $P_{\mathrm{AA}}>P_{\mathrm{BB}}=P_{\mathrm{AB}}$. Without loss of generality, we consider $P_{\mathrm{AA}}=1$ and define the sticking coefficient $r_{\mathrm{AB}}=P_{\mathrm{AB}} / P_{\mathrm{AA}}$. Lower sticking coefficients favor chain growth over branching.

In Ref. 34] we have shown that a crossover of the interfacial roughening from the Kardar-Parisi-Zhang (KPZ) to the KPZ with quenched disorder (KPZQ) is observed when $r_{\mathrm{AB}}$ is sufficiently small, i.e., when the $A$-patches are significantly stronger than the $B$-patches. For $r_{\mathrm{AB}} \ll$ 1 , the strong $A$-patches, promote growth along the poles, favoring the aggregation of $A A$ chains. These chains are likely to extend over long lateral regions. These long chains expose their $B$-patches and shield the access to any underlying $A$-patch, thus the probability of binding there is significantly lower. This is expected to have a similar effect to that of quenched noise 34 .

Note that the KPZ universality class is very robust [22, while KPZQ is only observed at the critical depinning transition [1, 56, 62. Nevertheless, for patchy
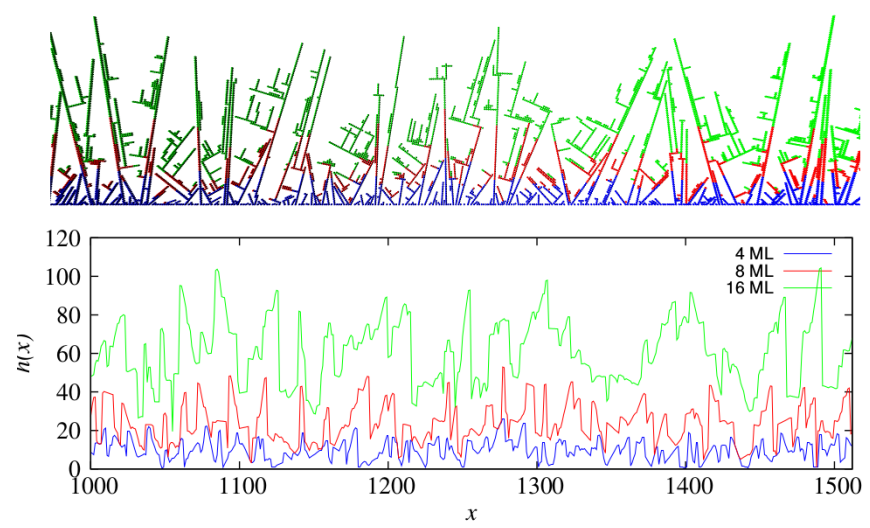

FIG. 11. Snapshot of a section of the aggregate (top) and height profile (bottom) after the adsorption of four (blue), eight (red), and 16 (green) monolayers of $2 A 2 B$ particles, with $r_{\mathrm{AB}}=0.01$. Results are for one single sample and a substrate of length $L=4096$.

particles with weak and strong bonds one finds remarkably an entire critical region of $r_{\mathrm{AB}}$ where KPZQ is observed. This is likely due to the balance of two competing mechanisms that keep the system at criticality. As $r_{\mathrm{AB}}$ decreases, the probability of binding to $B$-patches decreases and the growth of longer $A A$-chains increases. The longer these chains, however, the larger is the number of $B$-patches available for bonding, which compensates the decrease in the binding probability.

Recent experiments on aggregation of ellipsoids at the edge of an evaporating drop suggest that, for sufficiently large major-minor axis aspect ratio the interface is also in the KPZQ universality class 63. Together with these experiments, a discrete model was proposed to argue that KPZQ was driven by a colloidal Matthew effect 63. However, numerical simulations of the same model by Nicoli et al. 64] suggest a different interpretation of the theoretical results. Oliveira and Reis performed a careful statistical analysis of the correlation length and concluded that the differences observed in the growth and roughness exponents are due, instead, to a crossover to columnar growth 65. Although the results for the interface of the simple models appear to be settled the nature of the interface of the experimental system is still an open problem 63.

In order to proceed and to discard the possibility of columnar growth in the model of patchy particles with strong and weak bonds, we performed the analysis proposed in Ref. 65. Figure 11 shows a section of the aggregate and height profile for one sample with $r_{\mathrm{AB}}=0.01$. The time evolution of the height profile reveals no evidence of columnar growth. A more quantitative analysis is illustrated in Fig. 12 where we have plotted the scaling of the position $r_{0}$ of the first zero of the autocorrelation function with time. This position is expected to scale as,

$$
r_{0} \sim t^{1 / z}
$$




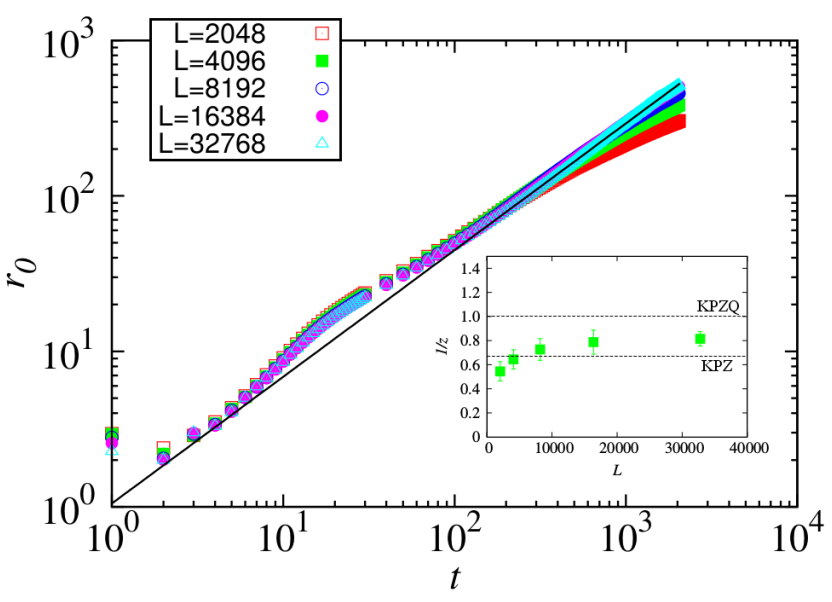

FIG. 12. Scaling of the first zero of the autocorrelation function with time for four different substrate lengths, $L=\{2048,4096,8182,16384,32768\}$, averaged over $\{20000,20000,10000,10000,2000\}$ samples. The inset depicts the size dependence of the slope $1 / z$, where error bars are given by the standard deviation of the local slope in the interval considered for the fitting. The dashed lines correspond to the expected value for KPZ (bottom) and KPZQ (top) universality classes.

where $z$ is the dynamic exponent. In spite of the strong finite-size effects, the size scaling of the slope (inset of the figure) indicates that the growth is consistent with the KPZQ universality class.

\section{FINAL REMARKS}

We reviewed our recent results on the kinetic roughening of interfaces of patchy particles and showed that the scaling of the interface depends strongly on the patchpatch correlations, the bond flexibility, and the strength of interactions. For particles with patch-patch correlations and bond flexibility we found two absorbing phase transitions that are, in general, of different nature. While the first transition is always continuous in the directed percolation universality class the second is either continuous or discontinuous depending on the bond flexibility. A scaling analysis of the size of the jump close to the tricritical flexibility suggests that the tricriticality is in the tricritical directed percolation universality class. For particles with weak and strong bonds we analyzed the kinetics of particles with two types of patches $(A$ and $B)$. When the strength of the $A$ and $B$ patches is similar, the interface roughness is in the Kardar-Parasi-Zhang universality class. However, for $A$-patches significantly stronger than the $B$-patches, the interface is in the universality class of Kardar-Parasi-Zhang with quenched disorder. This critical universality class is observed for an entire range of the relative strength of the interactions.

Beyond their theoretical interest, our findings have three consequences with practical applications. First, we have shown that the roughness of the interface can be controlled by the spatial distribution of patches or the relative strength of their interactions. Second, we revealed that sustained growth is only possible for certain patch arrangements. Third, the existence of an extended region of the parameter space where the critical KPZQ universality class is observed opens the possibility for an experimental realization of such systems using patchy colloids with weak and strong bonds.

\section{ACKNOWLEDGMENTS}

We acknowledge fruitful discussions with A. G. Yodh and T. J. Oliveira, as well as financial support from the Portuguese Foundation for Science and Technology (FCT) under Contracts nos. EXCL/FISNAN/0083/2012, PEst-OE/FIS/UI0618/2014, and $\mathrm{IF} / 00255 / 2013$.
[1] T. Halpin-Healy and Y.-C. Zhang, Phys. Rep. 254, 215 (1995).

[2] M. Vergeles, Phys. Rev. Lett. 75, 1969 (1995).

[3] J.-I. Wakita, H. Itoh, T. Matsuyama, and M. Matsushita, J. Phys. Soc. Jpn. 66, 67 (1997).

[4] H.-J. Kim, K. Park, and I.-M. Kim, Phys. Rev. E 64, 046103 (2001).

[5] M. Alava, M. Dubé, and M. Rost, Adv. Phys. 53, 83 (2004).

[6] O. Hallatschek, P. Hersen, S. Ramanathan, and D. R. Nelson, Proc. Natl. Acad. Sci. 104, 19926 (2007).

[7] H. Sakaguchi, Phys. Rev. E 82, 032101 (2010).

[8] K. A. Takeuchi and M. Sano, Phys. Rev. Lett. 104, 230601 (2010).

[9] M. A. C. Huergo, M. A. Pasquale, A. E. Bolzán, A. J. Arvia, and P. H. González, Phys. Rev. E 82, 031903 (2010).
[10] M. A. C. Huergo, M. A. Pasquale, P. H. González, A. E. Bolzán, and A. J. Arvia, Phys. Rev. E 84, 021917 (2011).

[11] K. A. Takeuchi, M. Sano, T. Sasamoto, and H. Spohn, Sci. Rep. 1, 34 (2011).

[12] K. A. Takeuchi and M. Sano, J. Stat. Phys. 147, 853 (2012).

[13] K. A. Takeuchi, Phys. Rev. Lett. 110, 210604 (2013).

[14] K. A. Takeuchi, J. Stat. Mech. 2014, P01006 (2014).

[15] S. N. Santalla, J. Rodríguez-Laguna, and R. Cuerno, Phys. Rev. E 89, 010401(R) (2014).

[16] A.-L. Barabási and H. E. Stanley, Fractal Concepts in Surface Growth (Cambridge University Press, Cambridge, 1995).

[17] P. Meakin, Fractals, scaling and growth far from equilibrium (Cambridge Univ Pr, Cambridge, 1998).

[18] G. Ódor, Rev. Mod. Phys 76, 663 (2004).

[19] M. J. Vold, J. Colloid Sci. 14, 168 (1959). 
[20] M. J. Vold, J. Colloid Sci. 695, 684 (1963).

[21] J. Blum and R. Schräpler, Phys. Rev. Lett. 93, 115503 (2004).

[22] M. Kardar, G. Parisi, and Y.-C. Zhang, Phys. Rev. Lett. 56, 889 (1986).

[23] C. S. Dias, N. A. M. Araújo, and M. M. Telo da Gama, Phys. Rev. E 87, 032308 (2013).

[24] A. B. Pawar and I. Kretzschmar, Macromol. Rapid Commun. 31, 150 (2010).

[25] S. Sacanna and D. J. Pine, Curr. Opin. Coll. Interf. Sci. 16, 96 (2011).

[26] F. Sciortino and E. Zaccarelli, Curr. Opin. Solid State Mater. Sci. 15, 246 (2011).

[27] Y. Iwashita and Y. Kimura, Soft Matt. 9, 10694 (2013).

[28] Y. Iwashita and Y. Kimura, Soft Matt. 10, 7170 (2014).

[29] O. Markova, J. Alberts, E. Munro, and P.-F. Lenne, Phys. Rev. E 90, 022301 (2014).

[30] S. Sokołowski and Y. V. Kalyuzhnyi, J. Phys. Chem. B 118, 9076 (2014).

[31] O. Pizio, S. Sokołowski, and Z. Sokołowska, J. Chem. Phys. 140, 174706 (2014).

[32] C. S. Dias, N. A. M. Araújo, and M. M. Telo da Gama, J. Chem. Phys. 139, 154903 (2013).

[33] C. S. Dias, N. A. M. Araújo, and M. M. Telo da Gama, Soft Matter 9, 5616 (2013).

[34] C. S. Dias, N. A. M. Araújo, and M. M. Telo da Gama, EPL 107, 56002 (2014).

[35] C. S. Dias, N. A. M. Araújo, and M. M. Telo da Gama, Phys. Rev. E 90, 032302 (2014).

[36] S. G. Alves, T. J. Oliveira, and S. C. Ferreira, arXiv:1409.2460 (2014).

[37] N. Geerts and E. Eiser, Soft Matt. 6, 4647 (2010).

[38] Y. Wang, D. R. Breed, V. N. Manoharan, L. Feng, A. D. Hollingsworth, M. Weck, and D. J. Pine, Nature 491, 51 (2012).

[39] M. E. Leunissen and D. Frenkel, J. Chem. Phys. 134, 084702 (2011).

[40] M. Henkel, H. Hinrichsen, and S. Lübeck, Nonequilibrium phase transitions (Springer, Bristol, 2008).

[41] S. Lubeck, Inter. J. Mod. Phys. B 18, 3977 (2004).

[42] R. Jullien and P. Meakin, J. Phys. A: Math. Gen. 22, L1115 (1989).

[43] C. J. Loweth, W. B. Caldwell, X. Peng, A. P. Alivisatos, and P. G. Schultz, Angew. Chem. Int. Ed. 38, 1808 (1999).

[44] S. Lübeck, J. Stat. Phys. 123, 193 (2006).
[45] P. Grassberger, J. Stat. Mech. 2006, P01004 (2006).

[46] J. M. Tavares, P. I. C. Teixeira, and M. M. Telo da Gama, Mol. Phys. 107, 453 (2009).

[47] J. M. Tavares, P. I. C. Teixeira, and M. M. Telo da Gama, Phys. Rev. E 80, 021506 (2009).

[48] J. M. Tavares, P. I. C. Teixeira, and M. M. Telo da Gama, Phys. Rev. E 81, 010501 (2010).

[49] J. M. Tavares, P. I. C. Teixeira, M. M. Telo da Gama, and F. Sciortino, J. Chem. Phys. 132, 234502 (2010).

[50] J. Russo, J. M. Tavares, P. I. C. Teixeira, M. M. Telo da Gama, and F. Sciortino, J. Chem. Phys. 135, 034501 (2011).

[51] J. Russo, J. M. Tavares, P. I. C. Teixeira, M. M. Telo da Gama, and F. Sciortino, Phys. Rev. Lett. 106, 085703 (2011).

[52] N. G. Almarza, J. M. Tavares, M. Simões, and M. M. Telo da Gama, J. Chem. Phys. 135, 174903 (2011).

[53] N. G. Almarza, J. M. Tavares, E. G. Noya, and M. M. Telo da Gama, J. Chem. Phys. 137, 244902 (2012).

[54] D. de Las Heras, J. M. Tavares, and M. M. Telo da Gama, Soft Matt. 8, 1785 (2012).

[55] J. M. Tavares, N. G. Almarza, and M. M. Telo da Gama, J. Chem. Phys. 140, 044905 (2014).

[56] Z. Olami, I. Procaccia, and R. Zeitak, Phys. Rev. E 49, 1232 (1994).

[57] Z. Csahók, K. Honda, and T. Vicsek, J. Phys. A: Math. Gen. 26, L171 (1993).

[58] H. Leschhorn, T. Nattermann, S. Stepanow, and L.-H. Tang, Ann. Physik 6, 1 (1997).

[59] L. A. N. Amaral, A.-L. Barabási, S. V. Buldyrev, S. T. Harrington, S. Havlin, R. Sadr-Lahijany, and H. E. Stanley, Phys. Rev. E 51, 4655 (1995).

[60] L. A. N. Amaral, A.-L. Barabasi, and H. E. Stanley, Phys. Rev. Lett. 73, 62 (1994).

[61] S. V. Buldyrev, A.-L. Barabási, F. Caserta, S. Havlin, H. E. Stanley, and T. Vicsek, Phys. Rev. A 45, R8313 (1992).

[62] L.-H. Tang and H. Leschhorn, Phys. Rev. A 45, R8309 (1992).

[63] P. J. Yunker, M. A. Lohr, T. Still, A. Borodin, D. J. Durian, and A. G. Yodh, Phys. Rev. Lett. 110, 035501 (2013).

[64] M. Nicoli, R. Cuerno, and M. Castro, Phys. Rev. Lett. 111, 209601 (2013).

[65] T. J. Oliveira and F. D. A. Aarão Reis, J. Stat. Mech. , P09006 (2014). 